\title{
Self-Neglect: A Hidden Killer
}

\author{
Mary Rose Day
}

School of Nursing and Midwifery, UCC

\section{Introduction}

Self-Neglect (SN) can be defined as:

"The inability (intentional or non-intentional) to maintain socially and culturally accepted standard of self-care with the potential for serious consequences to the health and wellbeing of the self-neglecters and perhaps even to their community" (Gibbons et al. 2006 p.16).

This definition is holistic and captures the intentional and choice factors as well as sociocultural influence of the behaviour and the potential negative impact of SN for the individual, their family and community. A key component of assessment by professionals is the individual's decision making capacity and ability to understand the consequences of their actions. Occasionally, distorted images and headlines appear in the media such as "A Systems Failure: Pensioner found dead due to Self-Neglect". There is a general outcry of public rage but the media are not interested in understanding why the vulnerable but competent adult who was self-neglecting is not rescued. A social media heading like this is a nightmare for professionals and services.

A range of interrelated factors contribute to health inequalities of older people such as socio-economic and material factors, psychosocial factors and lifestyle factors. Many of these are risk or contributory factors for SN in community-dwelling older adults. SN is associated with ageing, and the growth in people living to aged 80 years and over has the potential to increase the prevalence and incidence of SN. SN is a serious public health issue and it can occur along a continuum of severity and is frequently described as an older person's inability to provide for oneself the goods or services to meet basic needs. $\mathrm{SN}$ is poorly conceptualised and there is no universally accepted definition. Most states in the United States categorise SN as elder abuse and include it within conceptualisation of neglect and vulnerability. SN accounts for the majority of Adult Protective Services (APS) cases in the United States. Conversely, SN is not included in the definition of elder abuse in Ireland, UK, Europe and Australia as it does not occur within a relationship of trust. However procedural documents for referral to Elder Abuse Services (EAS) in Ireland have included those 'at risk of extreme self-neglect' and 18- 20\% of the referrals received are categorised as SN. Collectively, over the last 4 years, 1,739 SN referrals were made to services across the four Health Service Executive (HSE) areas in Ireland with under half 
coming from the South, the area the researcher is based. SN is difficult to detect and estimates may present only the tip of the "iceberg" as many cases go unreported or are unknown to services until they are chronic or life threatening.

\section{Physical, Psychosocial \& Environmental Dimensions: Hidden Factors in Self-Neglect}

A typical picture of people who severely self-neglect is one of very poor personal hygiene, malnourishment, living conditions of extreme squalor, vermin infested, with no running water or electricity and presence of a number of pets. Outsiders label such individuals as 'victims', 'reclusive' or 'eccentric' and many live alone and often refuse services.

Skills that enable individuals to live independently in the community are self-care tasks, activities of daily living (ADLs) (feeding, bathing, dressing, grooming, work, homemaking and leisure) and instrumental activities of daily living (IADLs) (doing housework, shopping, taking prescribed medication, managing finances, using telephone/technology or transport). Difficulties with handling finances, managing medication and using the telephone are more suggestive of problems in cognitive function, whereas difficulties with shopping, cooking, housework, laundry and transportation are more indicative of problems in physical function.

SN is associated with cognitive impairment, poor self-care, reduced physical function and is coupled with poor social support and poor income; self-neglecters are at serious risk for safe independent community living. Multiple psychosocial factors have been linked to self-neglect, such as alcohol/substance abuse, poor health, altered nutrition, dementia, depression, incontinence, pressure sores, impaired fragile skin, non-adherence to health advice or not taking prescribed medications or therapies. Alcohol/substance abuse in people who self-neglect is significantly associated with referral to APS services and more severe self-neglect. SN has been linked to poor social networks, poor family support, disconnectivity, service refusal and perceptions of poorer social resources were significantly related to increased risk for harm and reduced cognitive performance (Day et al. 2012). Individuals who SN are at higher risk for depression, isolation and abuse. SN is associated with higher rates of hospitalization, hospice care, nursing home placement, numerous physical health outcomes, early death and lower health status, including increased cardiovascular related mortality.

Higher SN prevalence is significantly linked to lower income. Many self-neglecters experience financial difficulties, suffer undue hardship and have annual incomes which are below the poverty line. Others appear to lack insight into how to manage their resources. Inadequate healthcare cover and inability to pay for needed formal support services can relate to eligibility and financial criteria. The current economic challenges and reductions 
in community resources could put older people at risk for SN.

According to Iris et al. (2010) environmental decline is a key factor in SN development more than personal decline related to health conditions. This argument challenges the conceptualization of SN as a medical syndrome. To date, no validated measures of SN have been embraced. Individuals that SN create many challenges for multidisciplinary teams in relation to choice, autonomy, self-determination and health and safety. Public health nurses are the main referrers of SN cases to EAS and have been identified by geriatricians as one of the most helpful services. A shared multidisciplinary team (MDT) approach to SN is very important. Understanding professional's perspectives of SN is very important. A dearth of research has explored the perspectives and experiences of those working with self-neglect cases in the community such as mental health workers, social workers, public health nurses and community nurses. No research so far has examined SN from a MDT perspective and in the Irish context; there has been little published research on SN. Thus the aim of this study is to investigate the physical, psychosocial and environmental dimensions of SN as perceived by multidisciplinary team members.

\section{Methodology}

A descriptive cross-sectional study will be conducted. The dimensions being reviewed are Physical and Psychosocial Aspects of SN (Physical Health Risks, SN and Mental Health and Social Networks and SN) and Environmental Aspects of SN (Physical and Personal Living Conditions, Pets, Social and Economic Issues). The review will inform the development of the questionnaire and will include a socio-demographic research developed tool. The survey questionnaire will be distributed online using survey monkey to a large national sample of multidisciplinary team members (public health nurses, community registered general nurses, community mental health nurses, practice nurses, senior social workers, elder abuse services, general practitioners, voluntary care workers) who are in contact with self-neglecting individuals. This will strengthen its potential for subsequent use in developing a risk assessment tool for self-neglect.

\section{Conclusion}

Ageing demographic changes means SN is a critical issue for society and policy makers. It is a complex multidimensional phenomenon, a hidden killer, a tragedy that occurs along a continuum of severity, and can be intentional or non-intentional. SN presents many challenges for professionals, services and society as a whole. It is poorly defined and conceptualised. Identifying multidisciplinary team members perspectives on the physical, social and environmental domains of self-neglect will provide information to advance risk 
assessment, decision-making, clinical guidelines and SN policy to better manage people who SN into the future. My research will inform interdisciplinary education and training on self-neglect.

Thanks to my supervisor Professor Geraldine McCarthy, Emeritus Professor, School of Nursing and Midwifery, UCC and colleagues for their continued support. 